\title{
SOLVING THE SOLAR AND ATMOSPHERIC NEUTRINO PROBLEMS WITH SUPERSYMMETRY
}

Talk given at the NATO Advanced Study Institute 2000, Cascais, Portugal, 26 June - 7 July, 2000.

\author{
J.C. ROMAO \\ Instituto Superior Técnico, Departamento de Física \\ Av. Rovisco Pais, 1, 1049-001 Lisboa, Portugal
}

\begin{abstract}
The simplest unified extension of the Minimal Supersymmetric Standard Model with bi-linear R-Parity violation provides a predictive scheme for neutrino masses which can account for the observed atmospheric and solar neutrino anomalies. Despite the smallness of neutrino masses Rparity violation is observable at present and future high-energy colliders, providing an unambiguous cross-check of the model.
\end{abstract}

\section{Introduction}

The recent announcement of high statistics atmospheric neutrino data by the SuperKamiokande collaboration [1] has confirmed the deficit of muon neutrinos, especially at small zenith angles, opening a new era in neutrino physics. Although there may be alternative solutions of the atmospheric neutrino anomaly [2] it is fair to say that the simplest interpretation of the data is in terms of $\nu_{\mu}$ to $\nu_{\tau}$ flavour oscillations with maximal mixing. This excludes a large mixing among $\nu_{\tau}$ and $\nu_{e}$ [1], in agreement also with the Chooz reactor data. On the other hand the persistent disagreement between solar neutrino data and theoretical expectations [3] has been a long-standing problem in physics. Recent solar neutrino data [4] are consistent with both vacuum oscillations and MSW conversions. In the latter case one can have either the large or the small mixing angle solutions, with a slight trend towards the latter [5].

Many attempts have appeared in the literature to explain the data. Here we review recent results [6] obtained in a model [7] which is a simple extension of the MSSM with with bilinear R-parity violation (BRPV). This model, despite being a minimal extension of the MSSM, can explain the 
solar and atmospheric neutrino data. Its most attractive feature is that it gives definite predictions for accelerator physics for the same range of parameters that explain the neutrino data.

\section{Broken $\mathbf{R}$-parity}

In the past most discussions of supersymmetric (SUSY) phenomenology assumed R-parity $\left(R_{P}\right)$ conservation where,

$$
R_{P}=(-1)^{2 J+3 B+L}
$$

This implies that SUSY particles are pair produced, every SUSY particle decays into another SUSY particle and that there is a $L S P$ that it is stable. But this is just an ad hoc assumption without a deep justification. In this talk we will review how $R_{P}$ can be broken, either spontaneously or explicitly, and discuss the most important features of these models [8].

\subsection{SPONTANEOUSLY BROKEN R-PARITY}

\subsubsection{The Original Proposal}

In the original proposal [9] the content was just the MSSM and the breaking was induced by

$$
\left\langle\tilde{\nu}_{\tau}\right\rangle=v_{L}
$$

The problem with this model was that the Majoron $J$ coupled to $Z^{0}$ with gauge strength and therefore the decay $Z^{0} \rightarrow \rho_{L} J$ contributed to the invisible $Z$ width the equivalent of half a (light) neutrino family. After LEP I this was excluded.

\subsubsection{A Viable Model for SBRP}

The way to avoid the previous difficulty is to enlarge the model and make $J$ mostly out of isosinglets. This was proposed by Masiero and Valle [10]. The content is the MSSM plus a few Isosinglet Superfields that carry lepton number. The model is defined by the superpotential [10],

$$
\begin{aligned}
W= & h_{u} u^{c} Q H_{u}+h_{d} d^{c} Q H_{d}+h_{e} e^{c} L H_{d} \\
& +h_{0} H_{u} H_{d} \Phi+\frac{\lambda}{3 !} \Phi^{3}+h_{\nu} \nu^{c} L H_{u}+h \Phi \nu^{c} S
\end{aligned}
$$

where the lepton number assignments are shown in Table (1). The spontaneous breaking of $\mathrm{R}$ parity and lepton number is driven by [10]

$$
v_{R}=\left\langle\tilde{\nu}_{R \tau}\right\rangle \quad v_{S}=\left\langle\tilde{S}_{\tau}\right\rangle \quad v_{L}=\left\langle\tilde{\nu}_{\tau}\right\rangle
$$


TABLE 1. Lepton number assignments.

\begin{tabular}{lccccc}
\hline Field & $L$ & $e^{c}$ & $\nu^{c}$ & $S$ & others \\
Lepton \# & 1 & -1 & -1 & 1 & 0 \\
\hline
\end{tabular}

The electroweak breaking and fermion masses arise from

$$
\left\langle H_{u}\right\rangle=v_{u} \quad\left\langle H_{d}\right\rangle=v_{d}
$$

with $v^{2}=v_{u}^{2}+v_{d}^{2}$ fixed by the $\mathrm{W}$ mass. The Majoron is given by the imaginary part of

$$
\frac{v_{L}^{2}}{V v^{2}}\left(v_{u} H_{u}-v_{d} H_{d}\right)+\frac{v_{L}}{V} \tilde{\nu_{\tau}}-\frac{v_{R}}{V} \nu_{\tau}^{\tilde{c}_{\tau}}+\frac{v_{S}}{V} \tilde{S_{\tau}}
$$

where $V=\sqrt{v_{R}^{2}+v_{S}^{2}}$. Since the Majoron is mainly an $S U(2) \otimes U(1)$ singlet it does not contribute to the invisible $Z^{0}$ decay width.

\subsubsection{Some Results on $S B R P$}

The SBRP model has been extensively studied. The implications for accelerator and non-accelerator physics have been presented before and we will not discuss them here [8]. In this talk we will only review the results for neutrinos. Neutrinos are massless at Lagrangian level but get mass from the mixing with neutralinos[11]. Neutrinos mix and the mixing is related to the the coupling matrix $h_{\nu_{i j}}$. This matrix has to be non diagonal in generation space to allow

$$
\nu_{\tau} \rightarrow \nu_{\mu}+J
$$

and therefore evading [11] the Critical Density Argument against $\nu^{\prime} s$ in the $\mathrm{MeV}$ range. In the $S M \mathrm{BBN}$ arguments [12] rule out $\nu_{\tau}$ masses in the range

$$
0.5 \mathrm{MeV}<m_{\nu_{\tau}}<35 \mathrm{MeV}
$$

We have shown [13] that SBRP models can evade that constraint due to new annihilation channels

$$
\nu_{\tau} \nu_{\tau} \rightarrow J J
$$

\subsection{EXPLICITLY BROKEN R-PARITY}

The most general superpotential $W$ with the particle content of the MSSM is given by [7] 


$$
W=W_{M S S M}+W_{R}
$$

where

$$
W_{M S S M}=\varepsilon_{a b}\left[h_{U}^{i j} \widehat{Q}_{i}^{a} \widehat{U}_{j} \widehat{H}_{u}^{b}+h_{D}^{i j} \widehat{Q}_{i}^{b} \widehat{D}_{j} \widehat{H}_{d}^{a}+h_{E}^{i j} \widehat{L}_{i}^{b} \widehat{R}_{j} \widehat{H}_{d}^{a}-\mu \widehat{H}_{d}^{a} \widehat{H}_{u}^{b}\right]
$$

and

$$
W_{R R}=\varepsilon_{a b}\left[\lambda_{i j k} \widehat{L}_{i}^{a} \widehat{L}_{j}^{b} \widehat{R}_{k}+\lambda_{i j k}^{\prime} \widehat{D}_{i} \widehat{L}_{j}^{a} \widehat{Q}_{k}^{b}+\lambda_{i j k}^{\prime \prime} \widehat{D}_{i} \widehat{D}_{j} \widehat{U}_{k}+\varepsilon_{a b} \epsilon_{i} \widehat{L}_{i}^{a} \widehat{H}_{u}^{b}\right]
$$

where $i, j=1,2,3$ are generation indices, $a, b=1,2$ are $S U(2)$ indices. To these we also have to add the soft supersymmetry breaking terms [6].

\section{Bilinear R-Parity Violation (BRPV)}

\subsection{THE MODEL}

The superpotential $W$ is given by

$$
W=\varepsilon_{a b}\left[h_{U}^{i j} \widehat{Q}_{i}^{a} \widehat{U}_{j} \widehat{H}_{u}^{b}+h_{D}^{i j} \widehat{Q}_{i}^{b} \widehat{D}_{j} \widehat{H}_{d}^{a}+h_{E}^{i j} \widehat{L}_{i}^{b} \widehat{R}_{j} \widehat{H}_{d}^{a}-\mu \widehat{H}_{d}^{a} \widehat{H}_{u}^{b}+\epsilon_{i} \widehat{L}_{i}^{a} \widehat{H}_{u}^{b}\right]
$$

while the set of soft supersymmetry breaking terms are

$$
\begin{aligned}
V_{\text {soft }} & =M_{Q}^{i j 2} \widetilde{Q}_{i}^{a *} \widetilde{Q}_{j}^{a}+M_{U}^{i j 2} \widetilde{U}_{i}^{*} \widetilde{U}_{j}+M_{D}^{i j 2} \widetilde{D}_{i}^{*} \widetilde{D}_{j}+M_{L}^{i j 2} \widetilde{L}_{i}^{a *} \widetilde{L}_{j}^{a}+M_{R}^{i j 2} \widetilde{R}_{i}^{*} \widetilde{R}_{j} \\
& +m_{H_{d}}^{2} H_{d}^{a *} H_{d}^{a}+m_{H_{u}}^{2} H_{u}^{a *} H_{u}^{a}-\left[\sum_{i} \frac{1}{2} M_{i} \lambda_{i} \lambda_{i}+\varepsilon_{a b}\left(A_{U}^{i j} \widetilde{Q}_{i}^{a} \widetilde{U}_{j} H_{u}^{b}\right.\right. \\
& \left.\left.+A_{D}^{i j} \widetilde{Q}_{i}^{b} \widetilde{D}_{j} H_{d}^{a}+A_{E}^{i j} \widetilde{L}_{i}^{b} \widetilde{R}_{j} H_{d}^{a}-B \mu H_{d}^{a} H_{u}^{b}+B_{i} \epsilon_{i} \widetilde{L}_{i}^{a} H_{u}^{b}\right)+h . c\right]
\end{aligned}
$$

The bilinear R-parity violating term cannot be eliminated by superfield redefinition. The reason is [14] that the bottom Yukawa coupling, usually neglected, plays a crucial role in splitting the soft-breaking parameters $B$ and $B_{i}$ as well as the scalar masses $m_{H_{d}}^{2}$ and $M_{L}^{2}$, assumed to be equal at the unification scale.

The BRPV model is a 1(3) parameter(s) generalization of the MSSM. It can be thought as an effective model showing the more important features of the SBRP-model at the weak scale. The mass matrices, charged and neutral currents, are similar to the SBRP-model if we identify

$$
\epsilon \equiv v_{R} h_{\nu}
$$

The model has the MSSM as a limit when $\epsilon_{i} \rightarrow 0$. 


\subsection{RADIATIVE BREAKING}

At $Q=M_{G U T}$ we assume the standard minimal supergravity unifications assumptions,

$$
\begin{aligned}
& A_{t}=A_{b}=A_{\tau} \equiv A, B=B_{2}=A-1, \\
& m_{H_{d}}^{2}=m_{H_{u}}^{2}=M_{L}^{2}=M_{R}^{2}=M_{Q}^{2}=M_{U}^{2}=M_{D}^{2}=m_{0}^{2}, \\
& M_{3}=M_{2}=M_{1}=M_{1 / 2}
\end{aligned}
$$

In order to determine the values of the Yukawa couplings and of the soft breaking scalar masses at low energies we first run the RGE's from the unification scale $M_{G U T} \sim 10^{16} \mathrm{GeV}$ down to the weak scale. For details see $[6,7]$.

\section{Tree Level Neutrino Masses and Mixings}

\subsection{NEUTRAL FERMION MASS MATRIX}

In the basis $\psi^{0 T}=\left(-i \lambda^{\prime},-i \lambda^{3}, \widetilde{H}_{d}^{1}, \widetilde{H}_{u}^{2}, \nu_{e}, \nu_{\mu}, \nu_{\tau}\right)$ the neutral fermions mass terms in the Lagrangian are given by

$$
\mathcal{L}_{m}=-\frac{1}{2}\left(\psi^{0}\right)^{T} \boldsymbol{M}_{N} \psi^{0}+\text { h.c. }
$$

where the neutralino/neutrino mass matrix is

$$
\boldsymbol{M}_{N}=\left[\begin{array}{cc}
\mathcal{M}_{\chi^{0}} & m^{T} \\
m & 0
\end{array}\right]
$$

with

$$
\mathcal{M}_{\chi^{0}}=\left[\begin{array}{cccc}
M_{1} & 0 & -\frac{1}{2} g^{\prime} v_{d} & \frac{1}{2} g^{\prime} v_{u} \\
0 & M_{2} & \frac{1}{2} g v_{d} & -\frac{1}{2} g v_{u} \\
-\frac{1}{2} g^{\prime} v_{d} & \frac{1}{2} g v_{d} & 0 & -\mu \\
\frac{1}{2} g^{\prime} v_{u} & -\frac{1}{2} g v_{u} & -\mu & 0
\end{array}\right] \quad ; \quad m=\left[\begin{array}{l}
a_{1} \\
a_{2} \\
a_{3}
\end{array}\right]
$$

where $a_{i}=\left(-\frac{1}{2} g^{\prime} v_{i}, \frac{1}{2} g v_{i}, 0, \epsilon_{i}\right)$. This neutralino/neutrino mass matrix is diagonalized by

$$
\mathcal{N}^{*} \boldsymbol{M}_{N} \mathcal{N}^{-1}=\operatorname{diag}\left(m_{\chi_{1}^{0}}, m_{\chi_{2}^{0}}, m_{\chi_{3}^{0}}, m_{\chi_{4}^{0}}, m_{\nu_{1}}, m_{\nu_{2}}, m_{\nu_{3}}\right)
$$

\subsection{APPROXIMATE DIAGONALIZATION OF MASS MATRICES}

If the $\not R_{p}$ parameters are small, then

$$
\xi=m \cdot \mathcal{M}_{\chi^{0}}^{-1} \quad \Rightarrow \quad \forall \xi_{i j} \ll 1
$$


one can find an approximate solution for the mixing matrix $\mathcal{N}$. Explicit solutions can be found in Ref. [6]. In leading order in $\xi$ the mixing matrix $\mathcal{N}$ is given by,

$$
\mathcal{N}^{*}=\left(\begin{array}{cc}
N^{*} & 0 \\
0 & V_{\nu}^{T}
\end{array}\right)\left(\begin{array}{cc}
1-\frac{1}{2} \xi^{\dagger} \xi & \xi^{\dagger} \\
-\xi & 1-\frac{1}{2} \xi \xi^{\dagger}
\end{array}\right)
$$

The second matrix above block-diagonalize $\boldsymbol{M}_{N}$ approximately to the form $\operatorname{diag}\left(m_{e f f}, \mathcal{M}_{\chi^{0}}\right)$

$$
m_{e f f}=-m \cdot \mathcal{M}_{\chi^{0}}^{-1} m^{T}=\frac{M_{1} g^{2}+M_{2} g^{2}}{4 \operatorname{det}\left(\mathcal{M}_{\chi^{0}}\right)}\left(\begin{array}{ccc}
\Lambda_{e}^{2} & \Lambda_{e} \Lambda_{\mu} & \Lambda_{e} \Lambda_{\tau} \\
\Lambda_{e} \Lambda_{\mu} & \Lambda_{\mu}^{2} & \Lambda_{\mu} \Lambda_{\tau} \\
\Lambda_{e} \Lambda_{\tau} & \Lambda_{\mu} \Lambda_{\tau} & \Lambda_{\tau}^{2}
\end{array}\right)
$$

The submatrices $N$ and $V_{\nu}$ in Eq. (22) diagonalize $\mathcal{M}_{\chi^{0}}$ and $m_{\text {eff }}$

$$
N^{*} \mathcal{M}_{\chi^{0}} N^{\dagger}=\operatorname{diag}\left(m_{\chi_{i}^{0}}\right) \quad ; \quad V_{\nu}^{T} m_{e f f} V_{\nu}=\operatorname{diag}\left(0,0, m_{\nu}\right),
$$

where

$$
m_{\nu}=\operatorname{Tr}\left(m_{e f f}\right)=\frac{M_{1} g^{2}+M_{2} g^{2}}{4 \operatorname{det}\left(\mathcal{M}_{\chi^{0}}\right)}|\vec{\Lambda}|^{2}
$$

\section{One Loop Neutrino Masses and Mixings}

\subsection{DEFINITION}

The Self-Energy for the neutralino/neutrino is

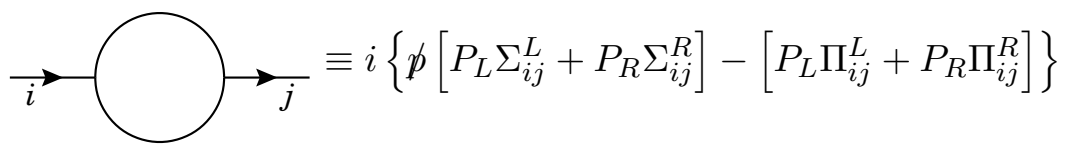

Then

$$
M_{i j}^{\text {pole }}=M_{i j}^{\overline{\mathrm{DR}}}\left(\mu_{R}\right)+\Delta M_{i j}
$$

with

$$
\Delta M_{i j}=\left[\frac{1}{2}\left(\Pi_{i j}^{V}\left(m_{i}^{2}\right)+\Pi_{i j}^{V}\left(m_{j}^{2}\right)\right)-\frac{1}{2}\left(m_{\chi_{i}^{0}} \Sigma_{i j}^{V}\left(m_{i}^{2}\right)+m_{\chi_{j}^{0}} \Sigma_{i j}^{V}\left(m_{j}^{2}\right)\right)\right]_{\Delta=0}
$$

where

$$
\Sigma^{V}=\frac{1}{2}\left(\Sigma^{L}+\Sigma^{R}\right) \quad ; \quad \Pi^{V}=\frac{1}{2}\left(\Pi^{L}+\Pi^{R}\right)
$$

and

$$
\Delta=\frac{2}{4-d}-\gamma_{E}+\ln 4 \pi
$$




\subsection{DIAGRAMS CONTRIBUTING}

In a generic way the diagrams contributing are
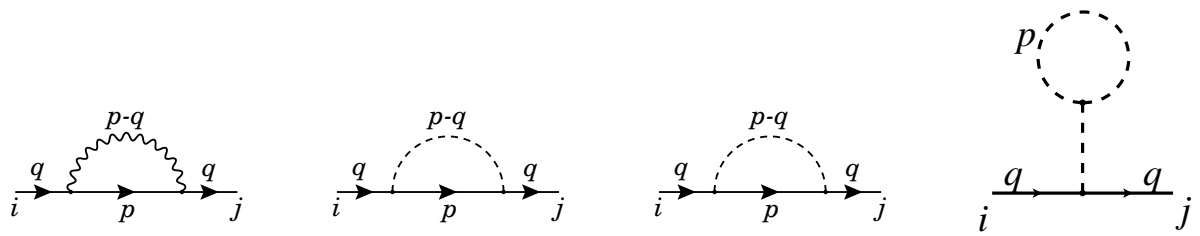

These diagrams can be calculated in a straightforward way. For instance the $W$ diagram in the $\xi=1$ gauge gives

$$
\begin{aligned}
& \Sigma_{i j}^{V}=-\frac{1}{16 \pi^{2}} \sum_{k=1}^{5} 2\left(O_{L j k}^{\mathrm{ncw}} O_{L k i}^{\mathrm{cnw}}+O_{R j k}^{\mathrm{ncw}} O_{R k i}^{\mathrm{cnw}}\right) B_{1}\left(p^{2}, m_{k}^{2}, m_{W}^{2}\right) \\
& \Pi_{i j}^{V}=-\frac{1}{16 \pi^{2}} \sum_{k=1}^{5}(-4)\left(O_{L j k}^{\mathrm{ncw}} O_{R k i}^{\mathrm{cnw}}+O_{R j k}^{\mathrm{ncw}} O_{L k i}^{\mathrm{cnw}}\right) m_{k} B_{0}\left(p^{2}, m_{k}^{2}, m_{W}^{2}\right)
\end{aligned}
$$

where $B_{0}$ and $B_{1}$ are the Passarino-Veltman functions, and $O^{\text {cnw }}, O^{\text {ncw }}$ are coupling matrices. Explicit expressions can be found in [6].

\subsection{GAUGE INVARIANCE}

When calculating the self-energies the question of gauge invariance arises. We have performed a careful calculation in an arbitrary $R_{\xi}$ gauge and showed [6] that the result was independent of the gauge parameter $\xi$.

\subsection{THE ONE-LOOP MASS MATRIX}

The one-loop corrected mass matrix is

$$
M^{1 L}=M_{\text {diag }}^{0 L}+\Delta M^{1 L}
$$

where

$$
M_{\text {diag }}^{0 L}=\mathcal{N} M_{N} \mathcal{N}^{T}
$$

Now we diagonalize the 1-loop mass matrix

$$
M_{\text {diag }}^{1 L}=\mathcal{N}^{\prime} M^{1 L} \mathcal{N}^{\prime T}
$$

Then the mass eigenstates are related to the weak basis states by

$$
\chi_{0}^{\text {mass }}=\mathcal{N}_{i \alpha}^{1 L} \chi_{0}^{\text {weak }}
$$


with

$$
\mathcal{N}^{1 L}=\mathcal{N}^{\prime} \mathcal{N}
$$

The usual convention in neutrino physics

$$
\nu_{\alpha}=U_{\alpha k} \nu_{k}
$$

is recovered in our notation as

$$
U_{\alpha k}=\mathcal{N}_{4+k, 4+\alpha}^{1 L}
$$

\subsection{SOLAR AND ATMOSPHERIC NEUTRINO PARAMETERS}

Assuming hierarchy in the masses $m_{\nu_{2}}$ and $m_{\nu_{3}}$ and neglecting $U_{e 3}$ (that has to be small) we write the usual two neutrino mixing angle as

$$
\sin ^{2}\left(2 \theta_{12}\right)=4 U_{e 1}^{2} U_{e 2}^{2}
$$

and

$$
\sin ^{2}\left(2 \theta_{13}\right)=4 U_{\mu 3}^{2}\left(1-U_{\mu 3}^{2}\right)
$$

\section{Results}

\subsection{MASSES AND MIXINGS}

The BRPV model produces a hierarchical mass spectrum for almost all choices of parameters. The largest mass can be estimated by the tree level value as shown in Fig. (1). As the figure shows, correct $\Delta m_{a t m}^{2}$ can be easily obtained by an appropriate choice of $|\vec{\Lambda}|$. The mass scale for the solar neutrinos is generated a 1 -loop level and therefore depends in a complicated way in the model parameters. This is shown in Fig. (2) where we have fixed the SUSY parameters. The parameter $\epsilon^{2} /|\vec{\Lambda}|$ is the most important in determining the solar mass scale, but some other parameters also play a role [6].

Now we turn to the discussion of the mixing angles. We have found that if $\epsilon^{2} /|\vec{\Lambda}| \ll 100$ then the 1 -loop corrections are not larger then the tree level results and the flavour composition of the 3rd mass eigenstate is approximately given by

$$
U_{\alpha 3} \approx \Lambda_{\alpha} /|\vec{\Lambda}|
$$

As the atmospheric and reactor neutrino data tell us that $\nu_{\mu} \rightarrow \nu_{\tau}$ oscillations are preferred over $\nu_{\mu} \rightarrow \nu_{e}$, we conclude that

$$
\Lambda_{e} \ll \Lambda_{\mu} \simeq \Lambda_{\tau}
$$




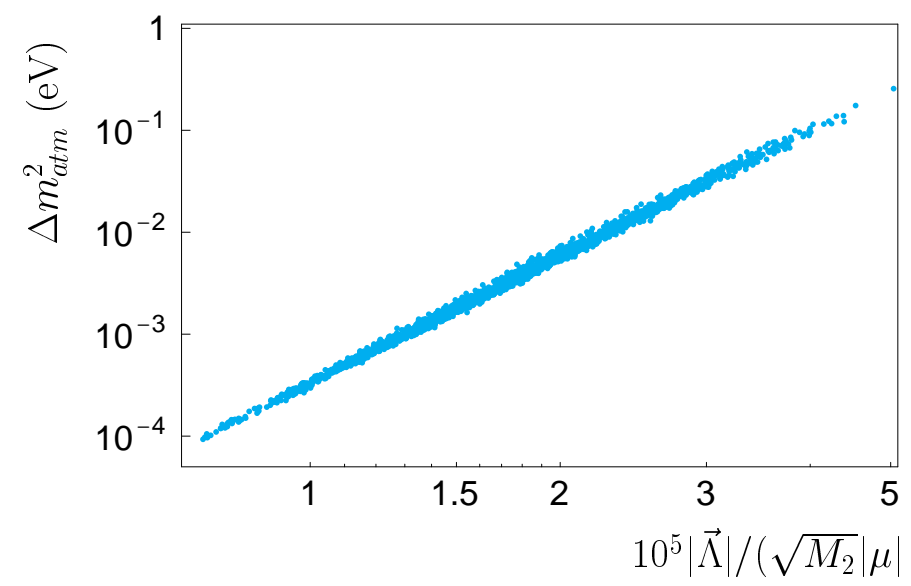

Figure 1. The atmospheric $\Delta m^{2}$ as function of $|\vec{\Lambda}| /\left(\sqrt{M_{2}} \mu\right)$

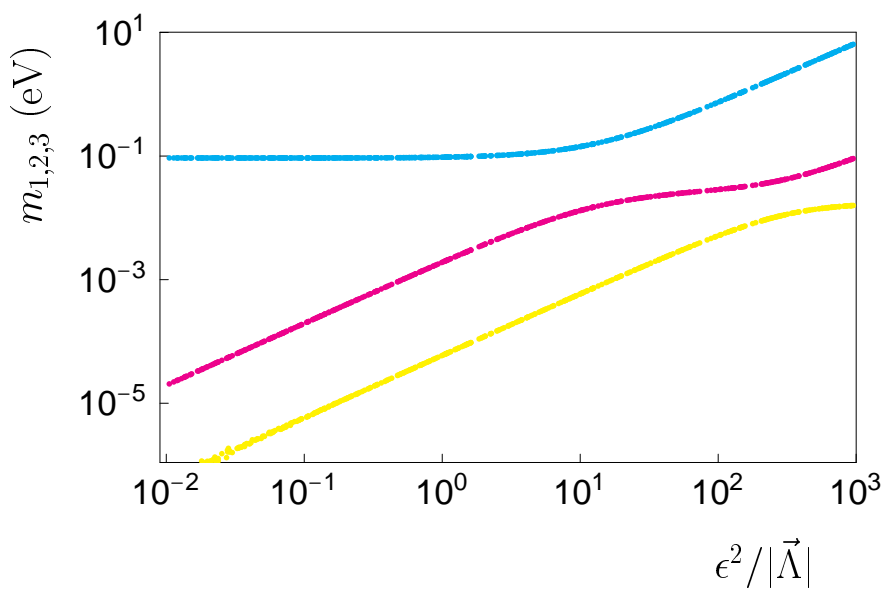

Figure 2. Neutrino masses as a function of $\epsilon^{2} /|\vec{\Lambda}|$

are required for BRPV to fit the the data. This is sown in Fig. (3).

For the solar angle the situation is more complicated and there are two cases to consider [6]. With the usual SUGRA assumptions, ratios of $\epsilon_{i} / \epsilon_{j}$ fix the ratios of $\Lambda_{i} / \Lambda_{j}$. Since atmospheric and reactor data tell us that $\Lambda_{e} \ll \Lambda_{\mu}, \Lambda_{\tau}$ in this case only the small angle solution can be obtained in the BRPV model as shown in Fig. (4 a). However we have shown that even a tiny deviation of from universality of the soft parameters at the GUT scale relaxes this constraint. In this case the ratio $\epsilon_{i} / \epsilon_{j}$ is not constrained and also large angle solutions can be obtained as shown in Fig. (4 b). 

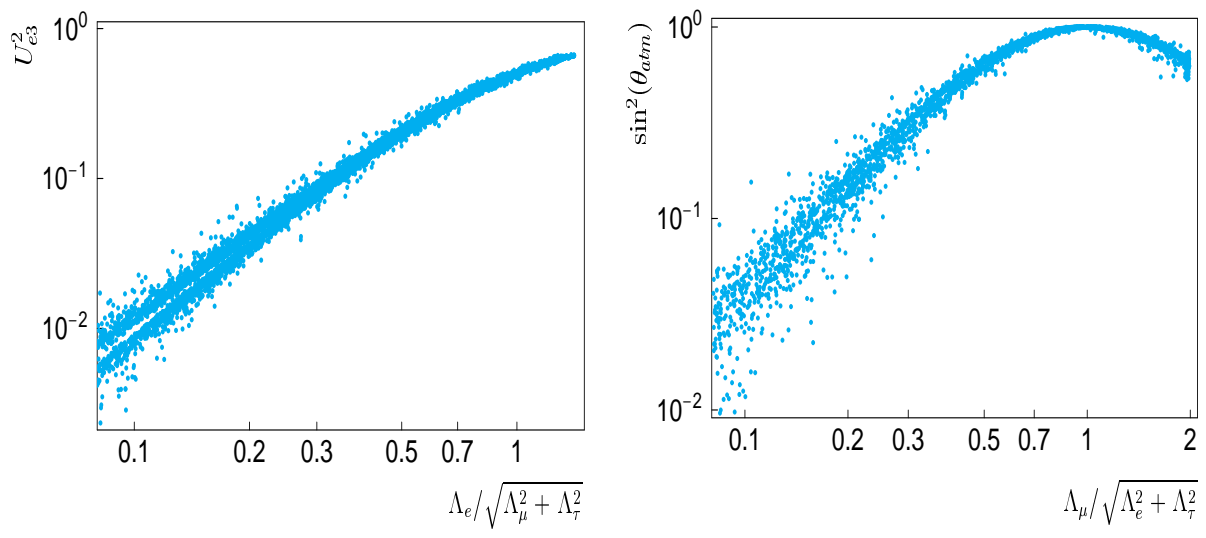

Figure 3. a) $U_{e 3}^{2}$ as a function of $\left|\Lambda_{e}\right| / \sqrt{\Lambda_{\mu}^{2}+\Lambda_{\tau}^{2}}$. b) Atmospheric angle as a function of $\left|\Lambda_{\mu}\right| / \sqrt{\Lambda_{\mu}^{2}+\Lambda_{\tau}^{2}}$.
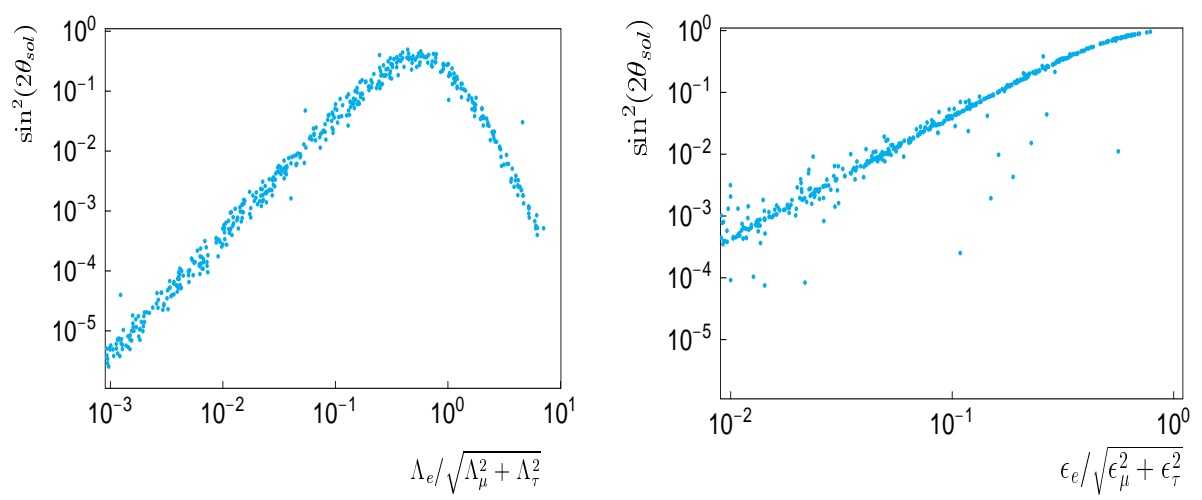

Figure 4. The solar angle as function of: a) $\left.\left|\Lambda_{e}\right| / \sqrt{\Lambda_{m u}^{2}+\Lambda_{\tau}^{2}} ; \mathrm{b}\right) \epsilon_{e} / \sqrt{\epsilon_{m u}^{2}+\epsilon_{\tau}^{2}}$

\subsection{CONSEQUENCES FOR THE ACCELERATORS}

One of the attractives of the BRPV model is that besides accommodating the solar and atmospheric neutrino data it can make definite predictions for accelerator physics. Then the model can be tested. As R-parity is violated, the neutralino is unstable. For this to have experimental consequences the neutralino has to decay well inside the detector. This is indeed the case as shown in Fig. (5 a). We have seen before that the ratios $\left|\Lambda_{i} / \Lambda_{j}\right|$ and $\epsilon_{i} /$ epsilon $_{j} \mid$ were very important in the choice of solutions for the neutrino mixing angles. What is exciting is that this ratios can be measured in accelerator experiments. In Fig. (5 b) we show the ratio of branching ratios for semileptonic LSP decays into muons and taus: $B R\left(\chi \rightarrow \mu q^{\prime} \bar{q}\right) / B R(\chi \rightarrow$ $\left.\tau q^{\prime} \bar{q}\right)$ as function of $\Lambda_{\mu} / \Lambda_{\tau}$. We can see that this is directly correlated with 

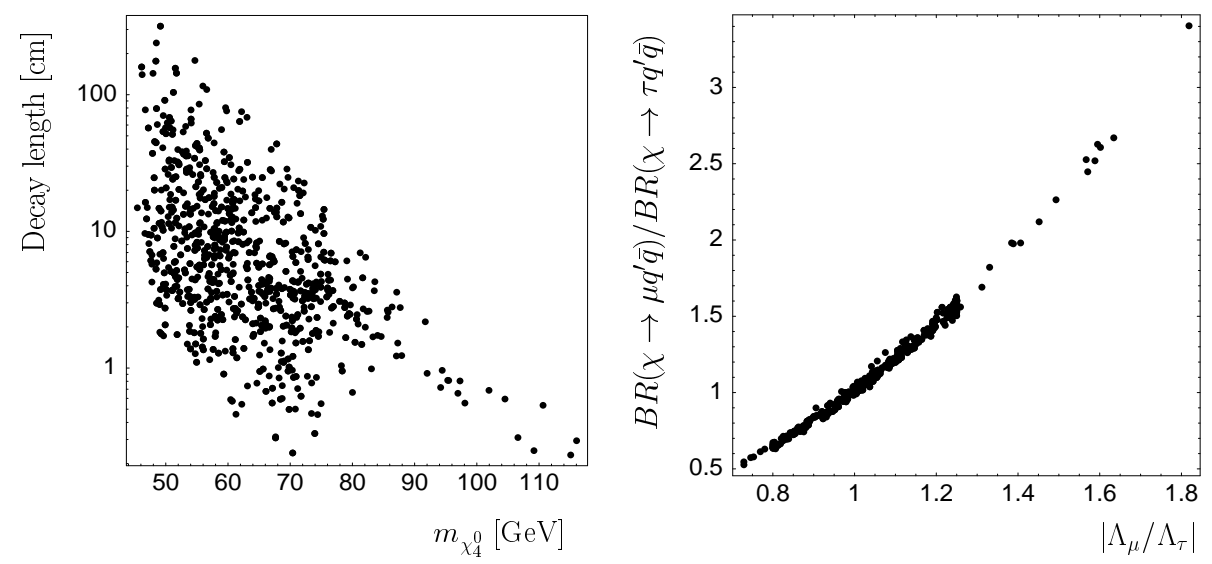

Figure 5. a) Neutralino decay length; b) $B R\left(\chi \rightarrow \mu q^{\prime} \bar{q}\right) / B R\left(\chi \rightarrow \tau q^{\prime} \bar{q}\right)$ as function of $\Lambda_{\mu} / \Lambda_{\tau}$
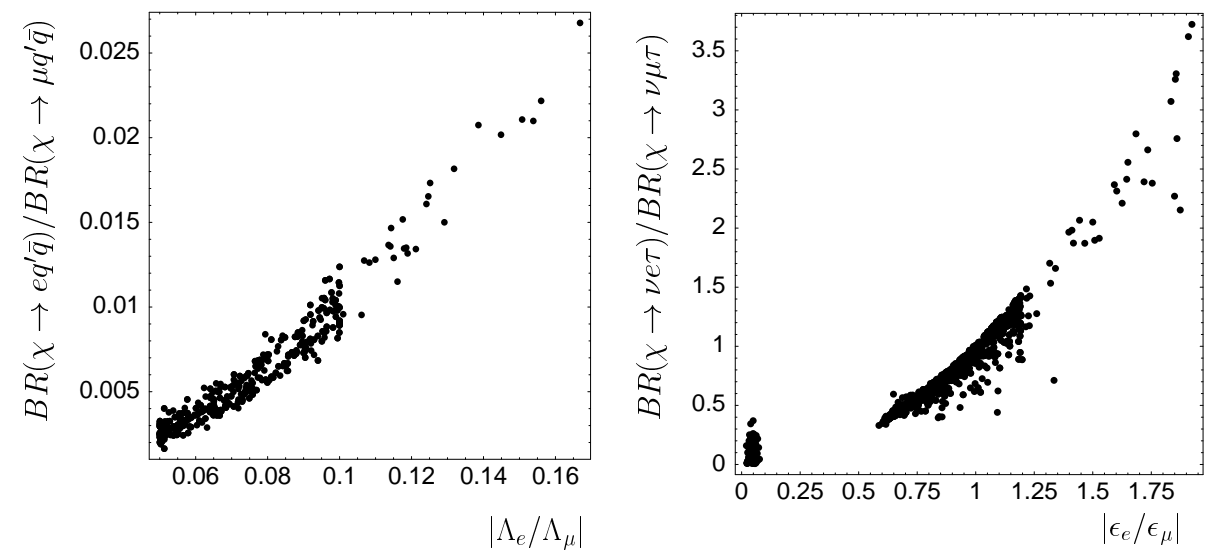

Figure 6. Ratio of branching ratios for semileptonic (a) and leptonic (b) LSP decays into $\mu$ and $\tau$ as functions of the BRPV parameters.

atmospheric angle. In Fig. (6 a) is shown the ratio of branching ratios for semileptonic LSP decays into muons and taus: $B R\left(\chi \rightarrow e q^{\prime} \bar{q}\right) / B R(\chi \rightarrow$ $\left.\mu q^{\prime} \bar{q}\right)$ as function of $\Lambda_{e} / \Lambda_{\mu}$. This is directly correlated with $U_{e 3}^{2}$. Finally in Fig. $(6 \mathrm{~b})$ we show the ratio of branching ratios for leptonic LSP decays into muons and taus: $B R(\chi \rightarrow \nu e \tau) / B R(\chi \rightarrow \nu \mu \tau)$ as function of $\left|\epsilon_{e} / \epsilon_{\mu}\right|$. This is directly correlated with solar angle.

\section{Conclusions}

We have shown that there is a viable model for SBRP that leads to a very rich phenomenology, both at laboratory experiments, and at present (LEP) 
and future (LHC, NLC) accelerators. Most of these phenomenology can be described by an effective model with explicit R-Parity violation. We have calculated the one-loop corrected masses and mixings for the neutrinos in a completely consistent way, including the RG equations and correctly minimizing the potential. We have shown that it is possible to get easily maximal mixing for the atmospheric neutrinos and both small and large angle MSW. We emphasise that the lightest neutralino decays inside the detectors, thus leading to a very different phenomenology than the MSSM. If the model is to explain solar and atmospheric neutrino problems then many signals will arise at future colliders. Thus the model can easily be proved wrong.

\section{References}

1. Fukuda, Y. et al. [Super-Kamiokande Collaboration] (1998), Phys. Rev. Lett. 81, 1562, hep-ex/9807003; see also hep-ex/9803006 and hep-ex/9805006.

2. Gonzalez-Garcia, M.C., Nunokawa H., Peres, O.L. and Valle, J.W. (1999) Nucl. Phys. B543, 3; Gonzalez-Garcia, M.C. et al. (1999), Phys. Rev. Lett. 82, 3202, hepph/9809531; Fornengo, N., Gonzalez-Garcia, M.C. and Valle, J. W. F. (1999), hepph/9906539; Barger, V. Learned, J.G., Pakvasa, S., and Weiler, T.J. (1999), Phys. Rev. Lett. 82, 2640.

3. Bahcall, J. N., Basu S. and Pinsonneault, M. H. (1998), Phys. Lett. B 433, 1.

4. Smy, M.B. (1999) "Solar neutrinos with SuperKamiokande," hep-ex/9903034.

5. For an updated discussion of solar and atmospheric neutrino data see the talk of M.C. Gonzalez-Garcia at the ICHEP 2000. An extended version of the talk can be found in Gonzalez-Garcia, M.C. and Peña-Garay, C. (1999) hep-ph/0009401.

6. Romão, J.C., Díaz, M.A., Hirsch, M., Porod, W. and Valle, J.W.F. (2000) Phys. Rev.D61, 071703 (2000); Hirsch, M., Díaz, M.A., Porod, W., Romão, J.C. and Valle, J.W.F. (2000), hep-ph/0004115 to appear in Phys. Rev.D,

7. Díaz, M.A., Romão, J.C. and Valle, J.W.F. (1998) Nucl. Phys. 524, 23.

8. For a review see e.g Romão, J. C. (1998) Lectures given at the 5th Gleb Wataghin School on High-Energy Phenomenology, Campinas, Brazil, 13-17 Jul 1998, hep$\mathrm{ph} / 9811454$.

9. Aulakh, C., Mohapatra, R. (1983) Phys. Lett. B119, 136; Santamaria, A., Valle, J.W.F. (1987) Phys. Lett. B195, 423; Santamaria, A., Valle, J.W.F. (1988) Phys. Rev. Lett. 60, 397.

10. Masiero, A. and Valle, J.W.F. (1990) Phys. Lett. B251, 273; Romão, J.C., Santos, C.A. and Valle, J.W.F. (1992) Phys. Lett B288, 311.

11. Nogueira, P., Romão, J.C. and Valle, J.W.F (1990) Phys. Lett B251, 142; Romão, J.C. and Valle, J.W.F. (1992) Nucl. Phys. B381, 87.

12. Bertolini, S. and Steigman, G. (1990) Nucl. Phys. B387, 193; Kawasaki, M. et al (1993) Nucl. Phys. B402, 323; Kawasaki, M. et al (1994) Nucl. Phys. B419, 105; Dodelson, S., Gyuk, G. and Turner, M.S. (1994) Phys. Rev. D49, 5068.

13. Dolgov, A.D., Pastor, S., Romão J.C. and Valle, J.W.F. (1997) B496, 24.

14. Díaz, M.A., (1998) Proceedings of the International Workshop on Physics Beyond the Standard Model: From Theory to Experiment (Valencia 97), Eds. Antoniadis, I., Ibañez, L.E. and Valle, J.W.F., World Scientific, pag. 188. 